\title{
La función de emparejamiento en los mercados de trabajo en transición: Revisión del caso checo
}

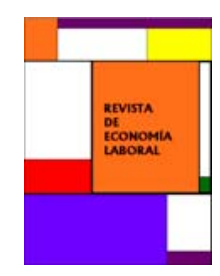

\author{
Pablo de Pedraza ${ }^{1}$ \\ Universidad de Salamanca
}

Recibido el 24 de abril de 2007; aceptado el 10 de octubre de 2007

\section{Resumen}

La literatura sobre la función de emparejamiento ha aumentado en los últimos quince años, siendo la República Checa uno de los principales protagonistas. Este trabajo realiza un repaso de dicha literatura. Después de la introducción, el apartado segundo analiza la importancia del enfoque de flujos en el análisis de los mercados de trabajo en transición y el significado de la función de emparejamiento. El tercer apartado repasa las principales conclusiones obtenidas por la literatura que utiliza la función de emparejamiento como un instrumento de análisis del funcionamiento mercado de trabajo checo.

Clasificación JEL: J6, J64, J69.

Palabras clave: Mercados de trabajo, transición, función de emparejamiento.

\begin{abstract}
The Czech Republic has figured prominently in matching literature over the last fifteen years. This type of literature has increased in parallel to the Central Eastern European transitions from planned to market economies. This paper makes a review of the matching literature that has focused on the Czech Republic. After the introduction, section two analyses both flows in transitional labour markets and the matching function. Section three reviews the main conclusions that can be found in the literature using the matching function as an instrument to analyse the working of the Czech labour market.
\end{abstract}

JEL Classification: J6, J64, J69.

Key words: Labour markets, transition, matching function.

1 Dirección para correspondencia: pablodepedraza@usal.es. Este trabajo se realizó durante una estancia de Investigación en el CERGE-EI Economic Institute de Praga. El autor quiere agradecer la ayuda recibida por Daniel Munich, Rafael Muñoz de Bustillo, Miguel Ángel Malo y los evaluadores anónimos.

(C) Revista de Economía Laboral 


\section{Introducción}

El objetivo del este trabajo es realizar una revisión de la literatura referente a la función de emparejamiento, más conocida como matching function, del mercado de trabajo checo. La intención es servir de guía a aquellos interesados en dicha función y que se enfrentan a su estudio por primera vez aunque se centren en el análisis de otros países.

Esta revisión literaria, referida solamente a un país, encuentra su justificación en que la función de emparejamiento checa ha sido sin duda la más estudiada por la literatura. Existen varios motivos que han llevado a los especialistas en los mercados de trabajo en transición a centrarse en el emparejamiento entre vacantes y desempleados en Chequia. En primer lugar, los bajos niveles de desempleo, sobre todo hasta 1998, a pesar del alto flujo de entrada en el desempleo durante la transición. En segundo lugar, el hecho de que Chequia y Eslovaquia fueran un mismo país hasta 1993 y compartieran instituciones, leyes y moneda $\mathrm{y}$, sin embargo, Eslovaquia tuviera niveles similares de desempleo al resto de países en transición, muy por encima de los de Chequia. Esta situación ha permitido comparar dos mercados de trabajo dejando fuera aspectos que suelen diferenciar a dos países y que, normalmente, de no tenerse en cuenta desvirtúan los resultados de la comparación y, de tenerse en cuenta, complican sobremanera el análisis. Una tercera razón se encuentra en la diversidad existente entre los 76 distritos checos en lo que se refiere a variables que determinan el funcionamiento del mercado de trabajo o son el resultado de éste, como el nivel de desempleo, el desempleo de larga duración, el nivel educativo de la población activa y la estructura económica heredada de los tiempos comunistas (ver anexo II). Por último, la abundante literatura sobre la función de emparejamiento checa y la necesidad de encontrar explicaciones a determinados resultados han dado lugar a toda una serie de literatura colindante que ayuda a la interpretación de los parámetros estimados. El estudio del caso checo, por tanto, ha permitido un mejor conocimiento de la información que de esta función se puede obtener.

Todo ello ha convertido a la República Checa, en ocasiones acompañada por la República Eslovaca, en una especie de laboratorio para el estudio de la matching function, su utilidad, sus limitaciones, las distintas metodologías para su estimación, las implicaciones que dichas metodologías tienen en las conclusiones que se pueden obtener, las correspondientes recomendaciones de política económica, la 
búsqueda de explicaciones para los resultados obtenidos y la aplicación de todo ello al estudio del resto de las economías, en transición o no.

Este trabajo se divide en los siguientes apartados. El apartado 2, sección 2.1, se centra en las economías en transición, la importancia de los flujos en el mercado de trabajo y, concretamente, en la importancia del flujo de salida del desempleo; la sección 2.2 trata sobre la interpretación de los parámetros estimados y las diferentes formas funcionales, especificaciones y estrategias de estimación que se pueden encontrar; la sección 2.3 emplaza la función matching en la teoría del desempleo de equilibrio; la sección 2.4 explica la importancia de la eficiencia del proceso de emparejamiento en el caso checo. El apartado 3 repasa la literatura existente sobre los dos enfoques que han estudiado el flujo de salida del desempleo. Por un lado, en la sección 3.1, los trabajos sobre las probabilidades individuales de abandonar el desempleo. Por otro, en la sección 3.2, aquellos que han estimado funciones agregadas de emparejamiento. El apartado 4 resume las conclusiones obtenidas.

\section{Flujo de salida del desempleo, función de emparejamiento y desempleo de equilibrio}

\subsection{Importancia del flujo de salida del desempleo en los procesos de transición}

Durante los últimos quince años ha habido un importante número de trabajos que han utilizado la función de emparejamiento, matching function o función matching, entre vacantes y desempleados como un instrumento de análisis del funcionamiento del mercado de trabajo y las heterogeneidades y fricciones existentes en él. El aumento de dicha literatura ha coincidido con los procesos de transición del comunismo al capitalismo en los países de Europa Central y Oriental y ha tenido a la República Checa como principal protagonista. En cierta manera, una de las causas del aumento de la literatura se encuentra en la importancia del flujo de salida del desempleo durante los procesos de transición. Dicha importancia, y, por tanto, la utilidad y relevancia de la función de emparejamiento, se puede explicar utilizando un cuadro de flujos (Gráfico 1) que esquematice el funcionamiento de los mercados de trabajo en transición.

El ritmo al que se van implementando las reformas de reestructuración y demolición del sector estatal determina el flujo de 
entrada en el desempleo representado por una línea roja. Muchos puestos de trabajo en sectores en declive como la industria pesada y el sector estatal desaparecen. La gran cantidad de trabajadores que empleaba el sector estatal comunista, hizo que muchos quedaran desempleados. Al mismo tiempo, se crean nuevos puestos en sectores en expansión como el sector servicios. El emparejamiento entre vacantes y desempleados produce el flujo de salida del desempleo al empleo que se representa con una línea azul oscuro.

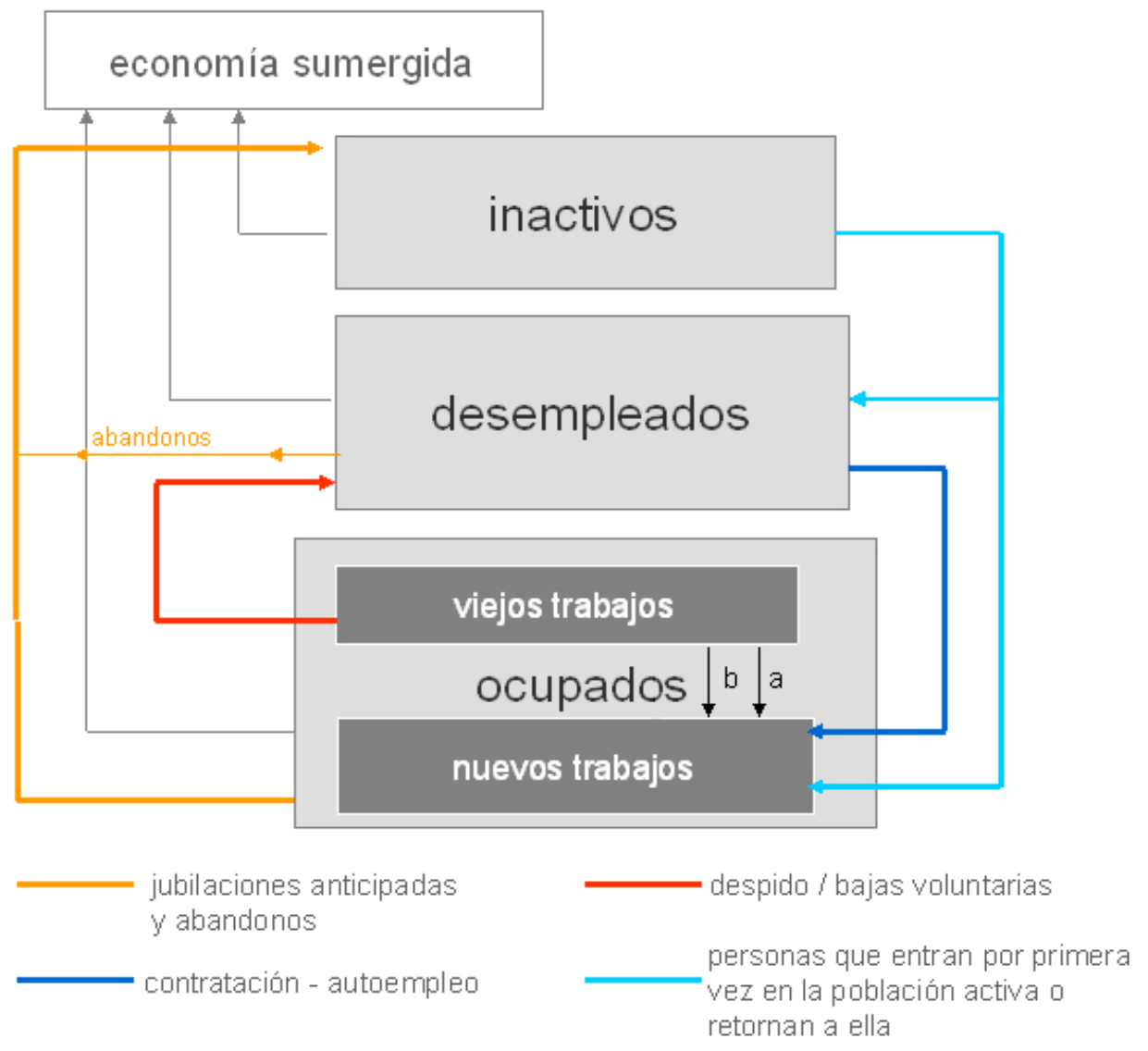

Gráfico 1.- Flujos en los mercados de trabajo en transición. 
Además, hay trabajadores que cambian de trabajo sin pasar por el desempleo y puestos de trabajo que sobreviven a la transición y continúan existiendo en la nueva economía de mercado, ambas situaciones se representan en el cuadro con las líneas negras a y b respectivamente. Por otra parte, también se produce un flujo de personas tanto empleadas como desempleadas que abandona la población activa, representado con la línea naranja. El flujo de entrada en la población activa, bien a la búsqueda de trabajo, bien a ocupar un puesto, se representa con la línea azul claro. Por último, no se puede olvidar la importancia que la economía sumergida tiene en las economías en transición, tanto empleados como desempleados que buscan trabajo y personas que no forman parte de la población activa pueden acabar trabajando en el sector informal de la economía. En este contexto, la economía sumergida puede actuar aumentando el efecto desincentivador que las prestaciones por desempleo y las prestaciones sociales producen en la búsqueda de un trabajo en el sector formal (Munich y Golusčak (2003)). Sin embargo, desgraciadamente, debido a la falta de datos sobre stock y flujo de trabajadores en el sector informal de la economía, la literatura de emparejamiento no ha cuantificado su importancia ni en el proceso de emparejamiento ni en la intensidad de la búsqueda.

Continuando con el enfoque de flujos en el mercado de trabajo, las conocidas como terapias de choque consisten en una demolición rápida del sector estatal. Los defensores de dichas terapias argumentan que una demolición rápida genera un aumento del flujo de entrada en el desempleo y, por tanto, una amplia reserva de trabajadores que permite el desarrollo de los nuevos sectores sin presiones salariales (Aghion y Blanchard (1993) y Ronald (2000)). Por otra parte, las terapias graduales consisten en una demolición más lenta de los viejos sectores. La defensa de estas últimas se basa en que una implementación lenta de las reformas, de manera flujos de entrada y salida se igualen, hace posible que paro y paro de larga duración aumenten menos reduciendo los efectos negativos del paro de larga duración en la eficiencia del proceso de emparejamiento. En otras palabras, se reduce la congestión en el mercado de trabajo. En este debate, terapia de choque versus terapia gradual, se encuentra el origen del aumento de la literatura sobre la matching function aplicada a las economías en transición, en general, y a la República Checa, en particular. Burda (1993) es el primer trabajo que utiliza la función de emparejamiento para analizar el flujo de salida del desempleo en economías en transición, utiliza 
datos de la República Checa ${ }^{2}$ y obtiene conclusiones sobre el ritmo al que las reformas deben ser aplicadas para evitar tanto congestión como presiones salariales.

\subsection{El significado de los parámetros estimados: externalidades y rendimientos a escala}

La función de emparejamiento ha sido utilizada en la literatura desde dos perspectivas. Por un lado, como una herramienta de modelización que, definida en términos de unas pocas variables, normalmente desempleo y vacantes, recoge las consecuencias que las heterogeneidades y fricciones del mercado de trabajo tienen en el equilibrio del modelo sin hacer referencia explícita a dichas heterogeneidades y fricciones. Por otro lado, precisamente como una herramienta cuya estimación permite el estudio de las heterogeneidades, fricciones y externalidades presentes en el mercado de trabajo. Las características de la función matching tienen importantes consecuencias en las propiedades de los equilibrios que se pueden derivar en los modelos que la utilizan como instrumento de modelización. A su vez dichas, características, pueden conocerse a través de la estimación de la función. En este sentido, toma especial relevancia la existencia o no de rendimientos constantes a escala en el proceso de emparejamiento entre vacantes y desempleados. En los trabajos de modelización se suele asumir que la matching function despliega rendimientos constantes a escala, asegurando así la existencia de un solo equilibrio en el modelo (ver gráfico 2). Sin embargo, los resultados de las estimaciones son muy diversos confirmando la existencia de rendimientos crecientes en muchas ocasiones, lo cual, es una condición necesaria para la existencia de más

\footnotetext{
2 Burda (1993) utiliza como marco teórico Pissarides (1992). Algunos trabajos anteriores a Burda (1993) que siguen enfoques parecidos aplicados a otras economías son por ejemplo, Pissarides (1986) que estimó la función de emparejamiento utilizando datos trimestrales del Reino Unido de 1967 a 1983; Blanchard y Diamond (1989) que utilizaron datos mensuales de Estados Unidos de 1968 a 1981; Layard, Nickell y Jackman (1991) que utilizaron datos trimestrales de Gran Bretaña de 1968 a 1988 y Van Ours (1991) que utilizó datos anuales de Holanda de1961 a 1987. Petrongolo y Pissarides (2001) buscan los orígenes de la función de emparejamiento en el pensamiento económico encontrándolos en los primeros autores que destacaron la importancia de las fricciones en el mercado de trabajo, concretamente se remonta al clásico de John Hicks La teoría de los salarios (1932 y 1963).
} 
de un equilibrio.

La función de matching agregada formaliza el proceso de emparejamiento entre vacantes y desempleados como aparece en la ecuación (1). H representa el número de contrataciones (es decir flujo de salida del desempleo, línea azul oscuro del gráfico 1) que depende, de forma análoga a un proceso de producción, de U (el número de desempleados) y V (el número de vacantes).

$$
H=\lambda m(U, V)
$$

El parámetro $\lambda$ representa el factor tecnológico y recoge las heterogeneidades y fricciones que tienen lugar en el proceso. Dicho parámetro es utilizado por Burda (1993) para obtener conclusiones sobre el ritmo al que deben ser implementadas las reformas para que flujos de entrada y salida se igualen, el desempleo aumente lo menos posible y se evite la congestión. Blanchard y Diamond (1989) señalan que la función de emparejamiento es análoga a una función producción y es consistente con la idea de que nuevos trabajos y trabajadores pueden diferir bien en su situación geográfica, bien en sus características y que, por ejemplo, regiones con una alta tasa de destrucción de trabajo pueden no coincidir con aquellas que tienen mayores tasas de creación de trabajo. Cambios en el parámetro $\lambda$ capturan cambios en las diferencias geográficas $u$ otras diferencias entre trabajadores y trabajos como las cualidades que los primeros tienen y las que son necesarias para ocupar un puesto. Es decir, el parámetro $\lambda$ captura lo que se ha denominado desajuste.

Se suele asumir que la función matching tiene una forma funcional Cobb-Douglass como la que aparece en la ecuación (2) con rendimientos constantes a escala.

$$
H_{t}=\lambda_{t} U_{t}^{\alpha} V_{t}^{\beta}
$$

Bajo el supuesto de rendimientos constantes a escala se cumple que $\alpha+\beta=1$. Ambos parámetros, las elasticidades de desempleados y vacantes con respecto al flujo de salida, reflejan aspectos como la intensidad de la búsqueda. Además, son una medida de las externalidades presentes en el proceso de emparejamiento porque la intensidad de la búsqueda de los desempleados tiene efectos en las probabilidades de encontrar trabajo de otros desempleados y en las probabilidades de las vacantes de ser ocupadas. Dicho de otra manera, en el proceso de emparejamiento se producen externalidades por varias 
razones: Lo que hacen las vacantes influye en el tiempo que tarda un desempleado en encontrar trabajo, dando lugar a externalidades positivas de vacantes en desempleados; de la misma manera, el tiempo que tarda una vacante en ser ocupada depende, no sólo de la búsqueda de candidatos por parte de las empresas, sino también del proceso de búsqueda de puestos de trabajo por parte de los desempleados, dando lugar a externalidades positivas de desempleados en vacantes; al mismo tiempo, lo que hacen unos desempleados influye en otros desempleados y lo que hacen unas vacantes influye en otras. En términos de la ecuación (2), a es una medida de la externalidad positiva de desempleados en vacantes y $a-1$ de la externalidad (negativa o congestión si $a<1$ ) de unos desempleados en otros. Por otro lado, $\beta$ mide la externalidad positiva de vacantes en desempleados (denominado en inglés como thick market effect) y $\beta$-1 la externalidad negativa de unas vacantes en otras.

No existen evidencias empíricas claras de que la función de emparejamiento tenga rendimientos a escala constantes, es decir, de que sea cierto que $\alpha+\beta=1$. Los resultados de los estudios existentes difieren incluso cuando la función matching es estimada para un mismo país. Como se verá en el apartado 3, la metodología de estimación juega un importante papel en las conclusiones que pueden obtenerse en este sentido.

Tampoco está claro que la forma funcional apropiada sea una Cobb-Douglas. Algunos estudios han estimado utilizando una translog (ecuación 3), forma funcional más flexible que la Cobb-Douglass y en la que esta está incluida ${ }^{3}$, obteniendo buenos resultados.

$$
\begin{aligned}
& \ln H_{t}=\beta_{0}+\beta_{1} \ln U_{t}+\beta_{2} \ln V_{t}+\frac{1}{2} \beta_{11}\left(\ln U_{t}\right)^{2} \\
& +\frac{1}{2} \beta_{22}\left(\ln V_{t}\right)^{2}+\beta_{12}\left(\ln V_{t}\right)\left(\ln U_{t}\right)
\end{aligned}
$$

La literatura también difiere en lo que se refiere a las variables explicativas, algunos trabajos han incluido otras variables además de desempleo y vacantes. Por ejemplo, según Coles y Smith (1994 y 1998), si los trabajadores inmersos en la búsqueda tienen información perfecta sobre las vacantes convocadas, entonces, hay una gran diferencia entre el stock de desempleados y vacantes y el flujo de nuevos desempleados y vacantes. En ese caso, el stock de desempleados y vacantes al principio

${ }^{3}$ La ecuación 3 es igual a la ecuación 2 si $\beta_{11}=\beta_{22}=\beta_{12}=0$. 
de un periodo, es decir, aquellos que ya estaban buscando en el periodo anterior, no intenta emparejarse entre sí en el nuevo periodo porque, de estar interesados en un emparejamiento, lo habrían hecho en el periodo anterior en el que ambos habían participado. Por tanto, en el proceso de emparejamiento el stock de desempleados intenta emparejarse con el flujo de nuevas vacantes y el stock de vacantes con el flujo de entrada en el desempleo. Este supuesto, denominado emparejamiento stock-flow, ha quedado reflejado en la especificación de la función de emparejamiento mediante la introducción del flujo de entrada en el desempleo como variable explicativa. Además, la función matching también se ha hecho dinámica mediante la introducción del valor retardado de la variable dependiente, flujo de salida del desempleo, como variable explicativa ${ }^{4}$. El resultado es una nueva especificación aumentada de la función de emparejamiento como la ecuación (4):

$$
\ln H_{t}=\beta_{0}+\beta_{H} \ln H_{t-1}+\beta_{U} \ln U_{t-1}+\beta_{V} \ln V_{t-1}+\beta_{N} \ln N_{t}
$$

El flujo de salida en el periodo t depende del flujo de salida en el periodo t-1 $\left(H_{t-1}\right)$, el nivel de desempleo $\left(U_{t-1}\right)$ y las vacantes $\left(V_{t-1}\right)$ al final del periodo t-1 y el flujo de entrada en el desempleo en el periodo $t$ $\left(N_{t}\right)$.

Por último, conviene resaltar que la literatura también es heterogénea en aspectos relacionados con la metodología y los datos. Por un lado, en la manera de abordar los problemas de autocorrelación y heterogeneidad no observada. Por otro, un problema muy común proviene de la dificultad de medir la cantidad de vacantes ${ }^{5}$.

\footnotetext{
${ }^{4}$ El valor retardado de la variable dependiente se incluye por dos razones. En primer lugar, el hecho de que la aceptación de un puesto puede preceder a la fecha en la que se registra el emparejamiento. En segundo lugar, el hecho de que la información sobre nuevas vacantes puede no difundirse instantáneamente a todos los desempleados. Ver Munich et al. (1998).

${ }^{5}$ En el caso de la República Checa, los trabajos utilizan datos de las oficinas de empleo de los distritos checos y, como suele ocurrir en otros países, aunque es obligatorio para las empresas registrar las vacantes que convocan, estas no tienen el incentivo de recibir una prestación como ocurre con los desempleados, además muchas vacantes quedan cubiertas instantáneamente y otras quedan registradas por largos periodos cuando, en realidad, ya no están convocadas.
} 


\subsection{Implicaciones de las características de la función de emparejamiento en un modelo de desempleo de equilibrio}

La discusión sobre los rendimientos a escala toma mayor importancia cuando la función de emparejamiento se utiliza como una herramienta de modelización. En caso de fuertes externalidades positivas puede haber rendimientos a escala crecientes, lo cual, es una condición necesaria para la existencia de más de un equilibrio y, por tanto, una justificación para la intervención estatal que lleve a la economía al equilibrio con menor desempleo.

Este aspecto puede explicarse fácilmente tomando como ejemplo una versión simplificada del modelo de desempleo de equilibrio de Pissarides $(1992,2000)$ en el que la función de emparejamiento, asumiendo rendimientos constantes a escala, es la piedra angular y el equilibrio es el resultado de las tres ecuaciones que se representan en el gráfico 2. Una curva salarial que sustituye la curva de oferta de trabajo convencional y tiene pendiente positiva: a mayor proporción de vacantes con respecto al número de desempleados $(\mathrm{v} / \mathrm{u}=\theta)$ mayor poder de negociación a favor de los trabajadores y mayores salarios. Una curva de creación de trabajo que sustituye a la curva de demanda de trabajo, teniendo en cuenta los gastos de contratación en los que incurren las empresas. Su pendiente es negativa: cuanto mayores son los salarios, la creación de puestos de trabajo es menos beneficiosa. Por último, la curva de Beveridge en la que está incluida la función de emparejamiento asumiendo rendimientos constantes a escala. Se define la curva de Beveridge como el lugar geométrico existente entre las tasas de paro y vacantes en relación a la población activa en una situación en la que el desempleo no varía, es decir, el flujo de entrada es igual al flujo de salida. El nivel de desempleo depende de ambos flujos, a su vez, la disponibilidad de vacantes afecta al flujo de salida, por tanto, existe una relación entre vacantes y desempleo. La variación del paro es la diferencia entre el flujo de entrada y el flujo de salida del paro. El primero depende del número de despidos y del nivel de empleo. El segundo depende del número de parados y de vacantes a través de la función de emparejamiento. En equilibrio, con una variación del paro igual a cero, la relación entre paro y vacantes es negativa: dado un proceso de búsqueda, cuanto mayor sea el número de vacantes, la probabilidad de encontrar un puesto de trabajo aumenta y menor será el número de parados. Si dicha probabilidad aumenta menos que proporcionalmente con el número de vacantes, la relación será convexa respecto del origen. Los puntos sobre la curva representan combinaciones de paro y vacantes donde la primera variable no cambia; 
los puntos por encima de la curva indican una mayor tasa de vacantes para cada tasa de paro, por lo que el desempleo disminuye; los puntos por debajo de la curva indican una menor tasa de vacantes para cada tasa de paro, por lo que el desempleo aumenta.

Salarios de equilibrio y proporción v/u

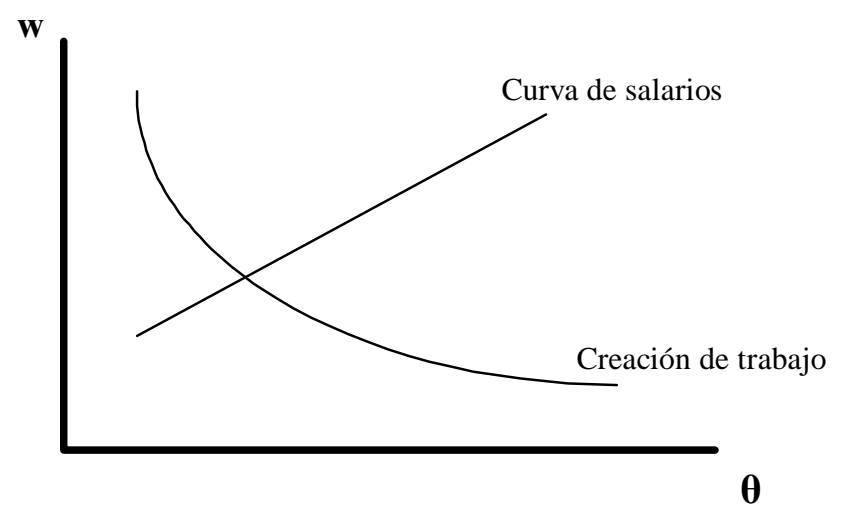

Vacantes y desempleo de equilibrio

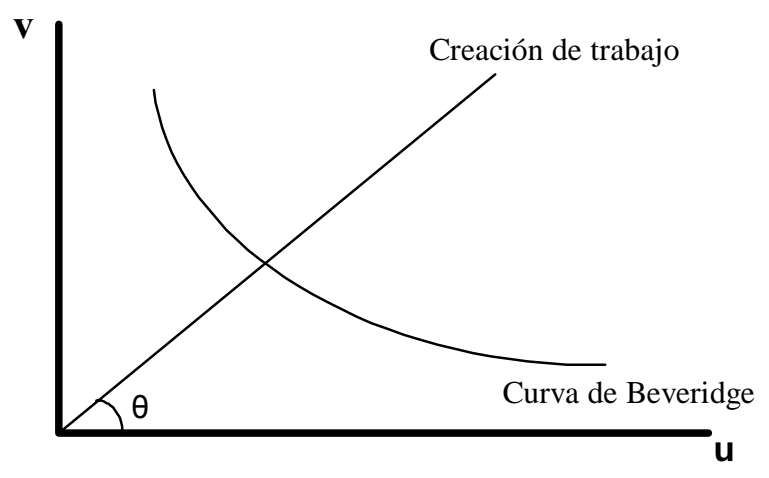

Grafico 2.- Desempleo de equilibrio

$\theta=\mathrm{v} / \mathrm{u}, \mathrm{w}$ : salarios, $\mathrm{u}$ : tasa de desempleo, v: tasa de vacantes.

El equilibrio resultante es único, estable y óptimo, lo cual no deja lugar para la intervención estatal en el proceso de emparejamiento. Sin embargo, en presencia de rendimientos crecientes a escala el equilibrio podría no ser único. La intervención estatal estaría justificada para llevar a la economía del equilibrio con alto nivel de desempleo al equilibrio con bajo nivel de desempleo. Una de las conclusiones a las que ha llegado la literatura que se repasa en el apartado 3.2, es que la forma funcional y el método de estimación influyen en las conclusiones que se pueden obtener en cuanto a los rendimientos a escala $\mathrm{y}$, por tanto, a las conclusiones sobre recomendaciones de política económica.

\subsection{Importancia de la función de emparejamiento en la transición Checa}

La economía Checa tuvo niveles de desempleo muy bajos hasta la crisis de 1997, después las tasas de paro crecieron, en parte debido al 
menor número de vacantes, en parte debido a la perdida de eficiencia en el proceso de emparejamiento. Dicho proceso observa en el gráfico 4 que puede ser interpretado en los términos que establece el gráfico 3 que, a su vez, resume tres tipos de shocks que pueden afectar a la curva de Beveridge.

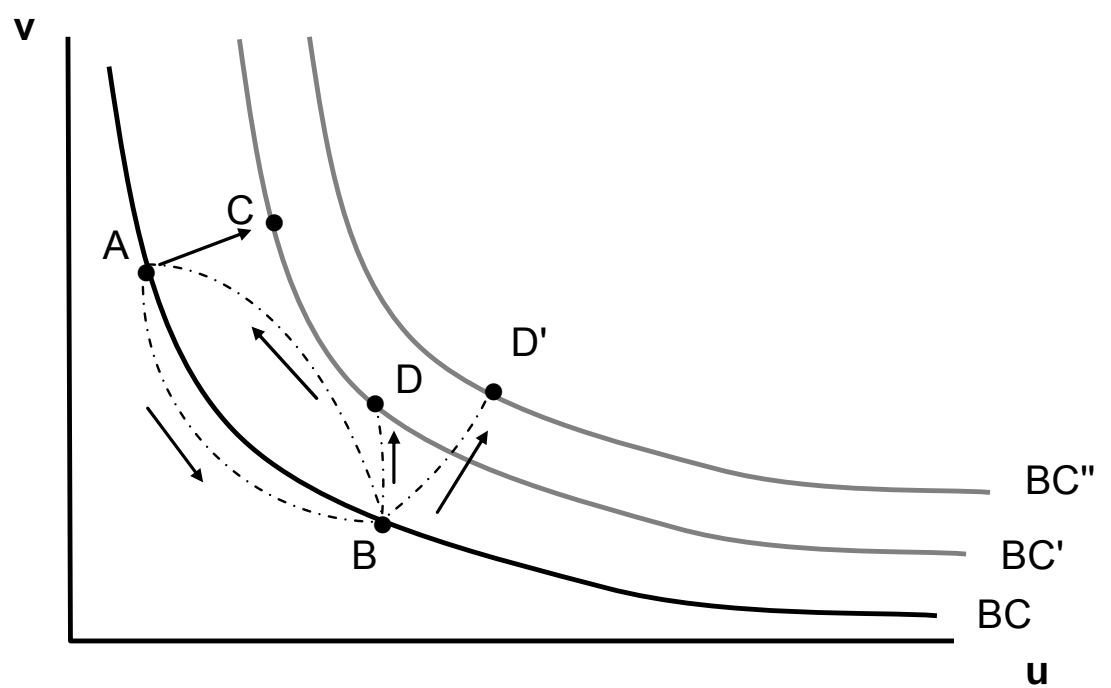

Gráfico 3.- Shocks en la Curva de Beveridge.

BC: Curva de Beveridge, u: tasa de desempleo, v: tasa de vacantes.

En primer lugar, shocks transitorios de demanda agregada ante los cuales se reduce el número de vacantes y se realizan despidos pasando la economía del punto $\mathrm{A}$ al punto $\mathrm{B}$ y una vez pasan los efectos del shock aumenta el número de vacantes, disminuye el paro y la economía vuelve al punto A. En segundo lugar, shocks de reasignación en los que desempleo y vacantes se mueven en la misma dirección, pasando del punto $\mathrm{A}$ al punto $\mathrm{C}$. Y por último, shocks transitorios de actividad agregada en presencia de histéresis en los que la economía pasa del punto A al B y, sin embargo, una vez transcurren los efectos del shock la economía no vuelve al punto A debido a una pérdida de eficiencia en el proceso de emparejamiento que puede venir dada por distintos motivos. Bien los efectos del desempleo de larga duración, bien 
presencia de desajustes sectoriales, porque los sectores en declive son distintos a los sectores en expansión, o desajustes educacionales, las habilidades y formación necesarias en los sectores nuevos son distintas a las que eran necesarias en los sectores en declive, o incluso desajustes geográficos, es decir, las vacantes en los sectores nuevos están siendo convocadas en distritos o regiones distintos a aquellos que se están liberando mano de obra.

El gráfico 4 representa los valores observados de desempleo y vacantes de la República Checa. Puede apreciarse un shock de reasignación durante los primeros años de la transición (1992-1996), un shock de actividad agregada de 1997 a 1999 y una pérdida de eficiencia en el proceso de emparejamiento una vez la economía recupera el crecimiento positivo. Algunos trabajos han utilizado el parámetro $\lambda$ de la ecuación (1) para estudiar el efecto de nuevas variables en la eficiencia del proceso de emparejamiento.

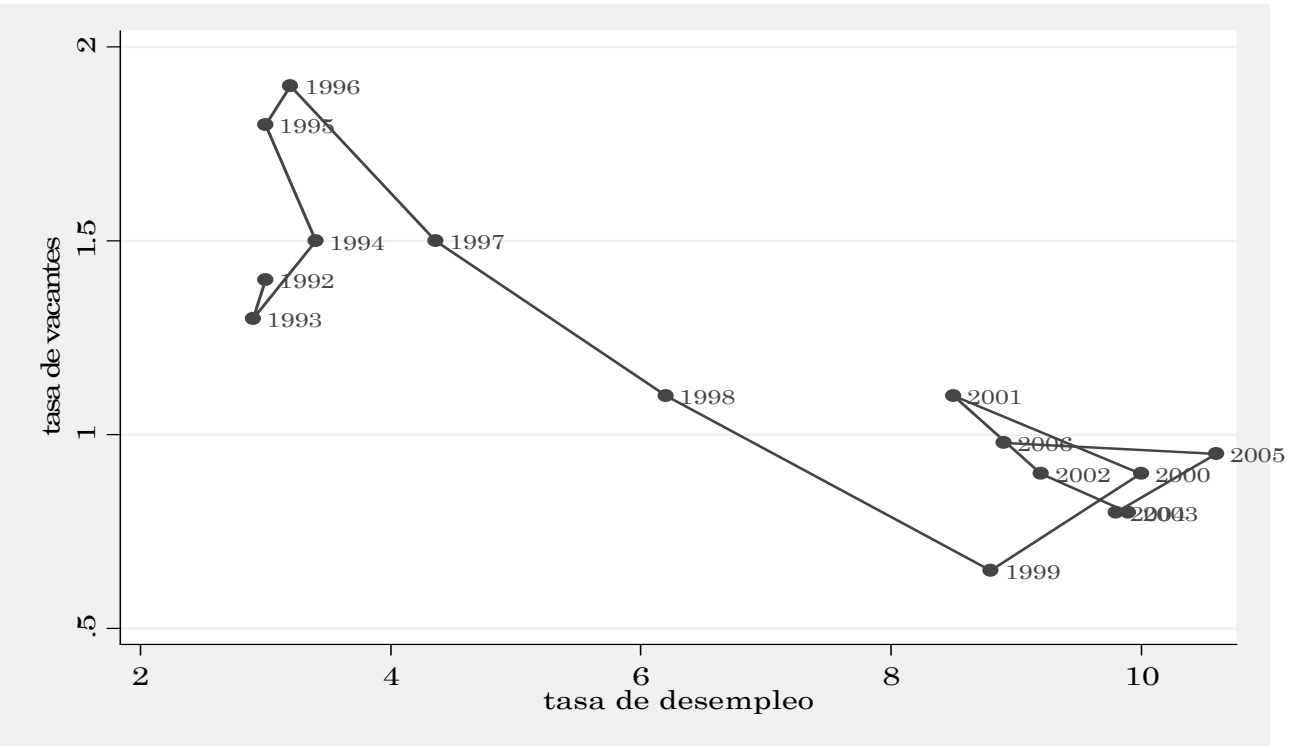

Gráfico 4.- Valores observados de desempleo y vacantes.

Como se apuntó en la introducción, existen importantes diferencias entre los distritos checos. Dichas diferencias han ido aumentando a lo largo de la transición. Por ejemplo, a finales de los 90 la tasa más baja de desempleo correspondía a Praga con un $4 \%$, la más alta a Ostrava con un $20 \%$. En lo que se refiere a la incidencia del paro 
de larga duración, el 11\% de los parados de Praga llevaba más de un año desempleado frente al $53 \%$ de otros distritos. Dichas diferencias tienen orígenes diversos, las distintas estructuras económicas heredades del comunismo, su evolución a lo largo de la transición, los niveles educativos de la población activa (ver Jurajda 2005) o la concentración de recursos en determinados distritos. Según Burda (1993) un $60 \%$ de las diferencias de paro entre los distritos puede explicarse con las diferencias en la eficiencia del emparejamiento entre vacantes y desempleados. Pedraza (2007) representa los valores observados de desempleo y vacantes de varios distritos concluyendo que las diferencias también existen en lo que se refiere al nivel de desajuste señalado en el gráfico 4.

\section{El estudio del flujo de salida: Las probabilidades individuales de abandonar el desempleo y la función de emparejamiento}

Por un lado, existen estudios que estiman las características individuales que influyen en las probabilidades de abandonar el desempleo, por otro, estudios que estiman funciones agregadas de emparejamiento. Los segundos dan información sobre la interacción entre desempleo y vacantes, los primeros pueden ser utilizados para la identificación de variables agregadas que pueden influir en el factor tecnológico de la función de emparejamiento, por ello, aunque este trabajo se refiere a la función agregada de emparejamiento, en el apartado 3.1 se incluye un pequeño repaso a este tipo de trabajos.

\subsection{Estudios sobre las variables que influyen en las probabilidades individuales de abandonar el desempleo}

En lo que se refiere a la estimación de las probabilidades individuales de abandonar el desempleo utilizando datos de la República Checa, los siguientes trabajos son los más relevantes. Teniendo en cuenta el excelente comportamiento del mercado de trabajo Checo hasta 1997, los altos niveles de desempleo en Eslovaquia y el hecho de que la diferencia viniera dada por las mayores tasas de salida del desempleo en Chequia, Ham et al. (1998) estudian la naturaleza de las causas de dicha diferencia y el papel que pueden jugar las políticas activas y pasivas de desempleo en aumentar o disminuir la duración del mismo. Para ello siguen a 3000 desempleados de Chequia y Eslovaquia desde octubre de 1991 y marzo de 1992 hasta que abandonan el 
desempleo o hasta julio de 1993. Utilizan datos semanales y calculan la probabilidad de abandonar el desempleo en la semana r. Ham et al. (1998) encuentran que, siendo la duración media del desempleo cuatro veces superior en Eslovaquia, casi una tercera parte de la diferencia puede explicarse por las diferencias observables en las condiciones de demanda (tasa de vacantes, producción industrial y nivel de desempleo del distrito), características demográficas y la estructura del empleo industrial. Los distintos comportamientos de empresas, individuos e instituciones pueden explicar los otros dos tercios. En ambas repúblicas el sistema de prestaciones por desempleo tiene un efecto negativo muy pequeño en aumentar la duración del desempleo y, por tanto, dichas prestaciones no han reducido la eficiencia del proceso de recolocación del factor trabajo en la transición. Pedraza (2007) también encuentra evidencias a nivel agregado que corroboran este resultado. Por último, encuentran que algunas variables demográficas como la educación no tienen un efecto claro en la duración del desempleo. Mientras en Eslovaquia los individuos con educación primaria tienen menos probabilidades de abandonar el desempleo que los que tienen educación secundaria y universitaria, en la República Checa el efecto de la educación sólo está claro en aquellos que no reciben prestaciones por desempleo. Ham et al. (1999) encuentran similares resultados aplicados a las mujeres.

Sorm y Terrel (1997, 1999a) usan microdatos de la Encuesta de Población Activa (EPA) checa de 1994 a 1998 y estiman logits multinomiales para las transiciones del desempleo a un trabajo y del desempleo al abandono de la población activa. En lo que se refiere a las transiciones a un puesto de trabajo, encuentran que no hay ningún grupo en términos de edad, nivel educativo, género o estado civil que tenga más dificultades que otros en encontrar trabajo. Por ejemplo, mientras las probabilidades de encontrar trabajo están positivamente relacionadas con la edad en 1994, en 1996 la edad no es significativa. Solamente encuentran una relación estable: encontrar trabajo es más difícil para los parados de larga duración, un mes más desempleado reduce las probabilidades de encontrar un trabajo en un $2.4 \%$.

Finta y Terrell (1997) usando datos de la EPA checa encuentran que las probabilidades de abandonar el desempleo decrecen con la edad y con la duración del desempleo, crecen con el nivel educativo y son mayores para las mujeres solteras que para las casadas y para los hombres casados que para los solteros. Lubyova y van Ours (1997) llevan a cabo un estudio similar usando datos de Eslovaquia llegando a la conclusión de que las probabilidades de abandonar el desempleo no están relacionadas con la edad, están negativamente relacionadas con 
el nivel de desempleo local, un punto porcentual más en el nivel de desempleo disminuye la probabilidades en un $42 \%$, y positivamente con el nivel educativo para los hombres, aquellos con educación universitaria tienen un $82 \%$ más de probabilidades de encontrar trabajo que aquellos con educación primaria, pero no para las mujeres y, además, son menores para los hombres solteros, un 34\%, que para los casados.

Sorm y Terrell (1998) analizan el impacto de las prestaciones por desempleo y las políticas activas de mercado de trabajo (PAMT) en la duración del desempleo. Encuentran que las PAMT han reducido la duración del desempleo de grupos cuyas experiencias tendían a ser más largas: mujeres, discapacitados, aquellos que ya han estado desempleados con anterioridad y los que tienen menor nivel educativo. También encuentran que el impacto de recibir prestaciones por desempleo en la duración de este es muy pequeño. Concluyen que ambos programas han sido efectivos en mejorar el funcionamiento del mercado de trabajo durante la transición, aumentando la admisibilidad social del desempleo y las probabilidades de encontrar trabajo de aquellos que se han beneficiado de estos programas.

Sorm y Terrell (1999b) no se centran en las probabilidades de abandonar el desempleo sino en la importancia de la movilidad del factor trabajo. Utilizando datos de 1994 a 1998 muestran que ha habido un importante movimiento hacia el sector financiero, el comercio y el turismo en detrimento de la agricultura y la industria y que más de la mitad de las personas que han cambiado de trabajo han cambiado también de sector de actividad. Los más jóvenes cambian más de trabajo y, aunque los que tiene un mayor nivel de estudios tienen más estabilidad en su puesto, sus probabilidades de encontrar trabajo cuando se encuentran en paro son mayores. Concluyen que el mercado de trabajo checo ha sido muy flexible y eficiente a lo largo de todo el proceso de recolocación que ha supuesto este cambio de estructura.

Las principales conclusiones que se pueden obtener de este tipo de trabajos son las siguientes. Las probabilidades de abandonar el desempleo suelen aumentar con el nivel educativo, disminuir con la duración del desempleo y difieren de unas regiones a otras. El sistema de prestaciones por desempleo checo no ha reducido la eficiencia del mercado de trabajo en el sentido de aumentar la duración del desempleo. En lo que se refiere a la comparación entre República Checa y Eslovaca, existen diferencias en cuanto al efecto de el nivel educativo, la edad y el estado civil.

Estudios sobre las probabilidades de abandonar el desempleo han sido también realizados para otras economías en transición. 
Bellman et al. (1995) utilizan datos de Alemania del Este, Abraham y Vodopivec (1993) de Slovenia, Micklewright y Nagy (1995 y 1997) de Hungría, Jones y Kato (1997) de Bulgaria, Dushi (1997) de Albania y Foley (1997) de Rusia. Las conclusiones con respecto a variables como edad, nivel educativo, sexo y estado civil también difieren de unos países a otros. El nivel educativo es la característica cuyo efecto positivo se repite con mayor frecuencia y el tiempo que dura la experiencia del desempleo aquella cuyo efecto negativo es más claro. Sin embargo, es difícil obtener conclusiones generalizables a todas las economías con respecto a características personales.

\subsection{Estimaciones de funciones de emparejamiento agregadas}

Este tipo de estudios aporta evidencias adicionales mediante la identificación de los factores de demanda (vacantes) y la interacción entre demanda y oferta. A lo largo de la literatura, se encuentran importantes contradicciones en lo que se refiere a los rendimientos a escala. Sabemos que mientras unas economías pueden dar lugar a rendimientos constantes, otras pueden dar lugar a rendimientos crecientes, incluso, una misma economía puede evolucionar de rendimientos crecientes a rendimientos decrecientes como en el caso de Alemania (ver Gross 1997). Sin embargo, las contradicciones también se pueden encontrar entre la literatura aplicada a un mismo país, en este caso, la República Checa: Mientras algunos concluyen que la función de emparejamiento checa tiene rendimientos constantes, otros encuentran que despliega rendimientos crecientes. Todo indica que, distintas metodologías de estimación pueden dar lugar a distintos resultados. Es decir, la falta de homogeneidad en los resultados puede deberse a diferencias metodológicas: la manera de especificar la función, introduciendo o no otras variables explicativas además de desempleo y vacantes; la forma funcional, Cobb-Douglas o translog; la frecuencia de los datos, anual, mensual o trimestral; el uso de datos de panel y otros aspectos como la autocorrelación o la heterogeneidad no observada que, de no ser tenidos en consideración, pueden sesgar los parámetros estimados. Como se puede observar en el siguiente repaso, las características técnicas de los trabajos tienen implicaciones en las conclusiones que se obtienen y, por tanto, en sus recomendaciones de política económica. Mientras la utilización de una forma funcional Cobb-Douglass estimada por MCO lleva a aceptar la existencia de rendimientos constantes $\mathrm{y}$, por tanto, a recomendaciones de política económica de no intervención estatal en el proceso de emparejamiento, realizar la misma estimación, usando los mismos datos pero aplicando 
algún método, bien primeras diferencias, bien desviaciones con respecto a la media, para eliminar la autocorrelación lleva a la conclusión de que existen rendimientos crecientes. En general, la literatura ha ido evolucionando de la estimación de funciones de emparejamiento sencillas, incluyendo sólo desempleo y vacantes como variables explicativas, utilizando la forma funcional Cobb-Douglas y estimando por MCO, a la inclusión de más variables explicativas, la utilización de otras formas funcionales más flexibles y otros métodos alternativos de estimación para corregir el sesgo que produce la agregación.

De cualquier manera, como apunta Svejnar (1999), cuando se utilizan datos de panel la forma funcional más frecuente es una CobbDouglas como la siguiente:

$$
\log O_{i, t}=c+\beta_{u} \log U_{i, t-1}+\beta_{v} \log V_{i, t-1}+\alpha_{i}+\lambda_{t}+\varepsilon_{i, t}
$$

donde $U_{i, t-1}$, y $V_{i, t-1}$ representan el número de desempleados y de vacantes al final del periodo $t-1, O_{i, t}$ representa el flujo de salida a puestos de trabajo, es decir, el número de emparejamientos entre vacantes y desempleados, la constante c captura la eficiencia del proceso de emparejamiento y $\alpha_{i}, \lambda_{t}$ y $\varepsilon_{i, t}$ representan características específicas de cada distrito o región que no varían en el tiempo, circunstancias de cada momento del tiempo y el conjunto de la parte no explicada respectivamente. Algunos trabajos han extendido la función incluyendo otras variables, por ejemplo, el número de personas que entran en el paro en el periodo actual t, el valor retardado de la variable dependiente, el gasto en políticas activas, los niveles educativos, la composición sectorial y otras que se verán a continuación.

El primer trabajo que utiliza la función de emparejamiento como herramienta para analizar los mercados de trabajo en transición es Burda (1993). Usa datos mensuales de los distritos Checos y Eslovacos y estima por mínimos cuadrados ordinarios (MCO) funciones de emparejamiento Cobb-Douglas simples, incluyendo sólo como variables explicativas desempleo y vacantes, y estáticas, no incluyendo el valor retardado de la variable dependiente. Llega a la conclusión de que existe una función de emparejamiento estable con rendimientos constantes a escala, cuyos coeficientes estimados para desempleados son el doble que los de las vacantes que, en el caso de Eslovaquia son muy pequeños y no significativos. Afirma que la existencia de una función de emparejamiento estable puede ser utilizada para el diseño de políticas en las economías en transición porque el crecimiento del sector 
privado no depende sólo de la velocidad a la que se pueden crear nuevas vacantes, sino de la eficiencia con la que puestos de trabajo y trabajadores se emparejen. Burda (1993) se centra en la velocidad óptima de transición (VOT), basándose en sus estimaciones de la función matching y su factor tecnológico, llega a la conclusión de que el ritmo al que deben implementarse las reformas, para mantener constante el nivel de desempleo, es el de una terapia gradual, intermedio entre la terapia de choque y las reformas lentas. Una reestructuración excesivamente rápida puede producir congestión, mientras que posponer las reformas reduce dicha congestión a costa de una menor creación de trabajo en los nuevos sectores. Conclusiones parecidas han sido obtenidas recientemente, incluyendo más años en el análisis y utilizando otra metodología por Jurajda y Terrell (2002 a y b). Burda (1993) asume que su análisis deja fuera importantes aspectos como el efecto del desempleo en el capital humano. Dichos aspectos han sido analizados en algunos de los trabajos que siguen.

Boeri (1994) utiliza datos regionales de la República Checa, Hungría, Polonia y Eslovaquia sobre periodos comprendidos entre 1991 y 1993. Estima por MCO funciones de emparejamiento Cobb-Douglas introduciendo desempleo y desempleo de larga duración por separado. Encuentra que, en general, el efecto de las vacantes es muy pequeño y que el impacto del desempleo de larga duración en el flujo de salida del desempleo es menor que el impacto del de corta duración. En cuanto a los rendimientos a escala, son bien decrecientes bien constantes.

Svejnar et al. (1995) usan datos anuales de 1992 y 1993 de Eslovaquia y Chequia y estiman por sección cruzada funciones de emparejamiento aumentadas, introduciendo variables demográficas y estructurales de los distritos y el gasto en políticas activas de mercado de trabajo (PAMT). Los coeficientes para desempleados son el doble en la República Checa que en la República Eslovaca y, de nuevo, los coeficientes para vacantes son muy bajos y en ocasiones no significativos. En cuanto a las PAMT, de acuerdo con los resultados obtenidos para la república Checa, un aumento en el gasto por desempleado del 1\% aumenta el flujo de salida en un 0,17\%. El efecto de dichas políticas en Eslovaquia no es estadísticamente significativo. Por último, la variación de la producción industrial es un importante determinante en el mayor flujo de salida checo.

Lubyova y van Ours (1994) utilizan datos mensuales de 1990 a 1992 de las regiones checas y eslovacas para estimar funciones de emparejamiento Cobb-Douglass para cada región. Buscan cambios estructurales y encuentran dos cambios negativos en la eficiencia de dos regiones checas y un cambio positivo en una región Eslovaca. 
Encuentran rendimientos crecientes en la mayoría de las regiones checas y dos regiones Eslovacas.

Boeri y Scarpetta (1995) utilizan datos mensuales de distritos checos, polacos, húngaros y eslovacos para estimar funciones de emparejamiento Cobb-Douglas aumentadas, mediante la introducción del empleo en la agricultura y una variable ficticia de la diversificación de la economía de cada distrito, para periodos comprendidos entre 1991 y 1994. Con la excepción de Eslovaquia, siempre encuentran rendimientos decrecientes.

Burda y Lubyova (1995) utilizan una combinación de datos mensuales y trimestrales de Chequia y Eslovaquia para medir la eficacia de las PAMT. Tienen en cuenta los efectos fijos de cada distrito estimando en diferencias con respecto a la media de todos los distritos e incluyen variables ficticias temporales. Sus conclusiones son que las PAMT tienen un efecto positivo y significativo en el flujo de salida del desempleo, que los coeficientes estimados para desempleo son muy altos mientras que los de vacantes son muy bajos, especialmente en Eslovaquia.

El objetivo de Boeri y Burda (1996) es también la cuantificación de los efectos de las PAMT, siguen una metodología similar y encuentran un efecto significativo pero pequeño. Estiman utilizando MCO y encuentran rendimientos ligeramente decrecientes.

Burda y Profit (1996), utilizando datos regionales y de distritos de 1992 a 1994 de la República Checa, ponen su atención en la gran dispersión existente en las tasas de desempleo de las regiones de Europa Central Oriental. Utilizando MCO no desechan la hipótesis de la existencia de rendimientos constantes a escala. Interpretan el aumento de la dispersión de las tasas de desempleo de la República Checa junto con la disminución del coeficiente de variación de las vacantes como una evidencia de la creciente importancia de los pequeños negocios y del desajuste. También llaman la atención sobre la importancia de las estructuras productivas heredadas de la Economía de Planificación Centralizada (EPC). Rechazan la estabilidad espacial de la función, resaltando la importancia de la movilidad del factor trabajo de unos distritos a otros y las mayores tasas de flujo de salida en los distritos que hacen frontera con Alemania. Encuentran la explicación de esos mayores flujos de salida en que muchas industrias manufactureras alemanas se han desplazado a la frontera checa para beneficiarse de menores costes laborales.

Profit (1996) utiliza datos de distritos checos de 1993 a 1994 y se centra en resolver los problemas de autocorrelación y heterocedasticidad en la estimación de la función. Demuestra que 
utilizando variables instrumentales Anderson-Hsiao se llega a la conclusión de que la función de emparejamiento checa despliega rendimientos crecientes a escala. El trabajo de Profit demuestra la importancia de la metodología de estimación en las conclusiones que se refieren a los rendimientos a escala. Los trabajos posteriores tienen en cuenta sus conclusiones en este sentido, encontrando rendimientos a escala crecientes. En consecuencia, algunos de los siguientes trabajos se centran en encontrar la fuente de dichos rendimientos.

Munich et al. (1998) vuelven a utilizar, como Burda (1993), el factor tecnológico de la función de emparejamiento y estiman las variables que influyen en él comparando Chequia y Eslovaquia. Utilizan datos de los distritos de ambos países de 1991 a 1996 y de 1991 a 1994 respectivamente. A diferencia de los anteriores trabajos, utilizan una forma funcional translog como la que aparece en la ecuación (4); introducen de forma separada los desempleados que entran en el desempleo en el periodo actual y los que ya estaban desempleados; tienen en cuenta en la estimación los distintos tamaños de los distritos, la endogeneidad de las variables explicativas y la heterogeneidad no observada. Rechazan la existencia de rendimientos constantes a escala. La suma de coeficientes de vacantes y desempleados oscilan entre $2.5 \mathrm{y}$ 3.5 en Chequia y entre 0,5 y 2,8 en Eslovaquia que evoluciona hacia rendimientos crecientes a medida que avanza la transición. Ambos, vacantes y desempleados, juegan un papel más importante en la República Checa que en Eslovaquia, aunque los desempleados han jugado un papel importante en ambas.

Profit (1999) utilizando datos de 1991 a 1994 llega a la conclusión de que una función de emparejamiento propiamente especificada y estimada consistentemente teniendo en cuenta la autocorrelación, la heterocedasticidad y la validez de los instrumentos utilizados da lugar a elasticidades flujo de salida con respecto a vacantes y desempleados que implican rendimientos crecientes a escala. Los estudios existentes, a excepción de los dos últimos, habían fallado en detectar este efecto. Por tanto, corroborada la existencia de rendimientos crecientes y los interpreta como el resultado de la competición existente entre las empresas estatales y el emergente sector privado por la mano de obra cualificada. Concluye que dicha competición da lugar a un aumento de los cambios de trabajo y que la intensidad en la búsqueda de los trabajadores empleados es la fuente de los rendimientos crecientes. La interpretación de los rendimientos a escala de Profit (1999) está en contradicción con uno de los supuestos de Burda (1993) que asumía que en el contexto de la transición la búsqueda de trabajo de los que ya ocupaban un puesto trabajo no era 
importante. También está en contradicción con Munich et al. (1998) cuyos resultados destacaban el importante papel desempeñado por los desempleados. Desgraciadamente no existe información sobre los empleados que buscan trabajo y el efecto de su búsqueda en la función de emparejamiento es difícil de inferir. Ante dicha falta de datos, Pedraza (2007) obtiene conclusiones sobre el papel que juegan los empleados en la función matching introduciendo como variables explicativas del factor tecnológico características que se refieren a la población activa en su conjunto y no sólo de los desempleados, encontrando que ambos tipos de variables son significativas.

Profit y Sperlich (2004) ponen atención en la variación de los rendimientos a escala entre regiones y encuentran que guarda relación con algunas características de estructura económica como la proporción de empleo agrícola, industrial y en el sector servicios y con las PAMT. Dejan para investigaciones futuras el papel que juega el nivel educativo de total de desempleados. Pedraza (2007) analiza el papel de la educación pero, tomando el nivel educativo del conjunto de la población activa.

Por último, Pedraza (2007), partiendo de los trabajos de Burda (1993) y Munich (1998), realiza una estimación en dos fases utilizando datos mensuales de los distritos checos de 1992 a 2000. En la primera fase pone atención en la existencia o no de rendimientos constantes a escala y, en la segunda, en la estimación de las características de los distritos que influyen en el factor tecnológico de la función, buscando la fuente de los rendimientos a escala. En la primera fase lleva a cabo varias especificaciones de la función y varias metodologías de estimación, llegando a la conclusión de que siempre que se tiene en cuenta la heterogeneidad no observada se obtienen rendimientos crecientes a escala. En lo que se refiere a la segunda fase encuentra evidencias de que todos los miembros de la población activa, y no sólo los desempleados, influyen en el proceso de emparejamiento y, por tanto, de que una de las fuentes de los rendimientos crecientes puede ser la señalada por Profit (1999). Sin embargo, dicha fuente no es la única ya que también encuentra evidencias del importante papel jugado por los desempleados, fundamentalmente a través del autoempleo en el sector comercial. Además, la población activa Checa, como indicaban Sorm y Terrel (1999 a y b), ha sido muy eficiente en cambiar de unos sectores a otros, según los resultados obtenidos por Pedraza (2007) dicha eficiencia ha venido dada por los trabajadores con educación secundaria y universitaria. Pedraza (2007) también corrobora algunas de las conclusiones de los trabajos anteriores como el efecto positivo que tiene sobre el flujo de salida la proximidad a la frontera con Alemania. 
En el anexo I se ofrece un cuadro que resume las principales características de los trabajos que se han revisado en esta sección.

$\mathrm{Al}$ utilizar la función matching como herramienta de modelización teórica, se suele asumir que tiene rendimientos constantes a escala; sin embargo, las contradicciones existentes en la literatura empírica que estima funciones de emparejamiento agregadas no permiten obtener conclusiones sobre lo acertado de dicho supuesto. En lo que se refiere a las estimaciones aplicadas a Chequia, cuando se utiliza como método de estimación los MCO y se usa una forma funcional Cobb-Douglass, se llega a la conclusión de que existen rendimientos constantes. Sin embargo, cuando se utilizan métodos de estimación que tienen en cuenta aspectos como la heterogeneidad no observada o la existencia de heterocedasticidad y/o se estima utilizando formas funcionales más flexibles, como una translog, se llega a la conclusión de que existen rendimientos crecientes a escala. Por tanto, por un lado, la agregación introduce heterogeneidades que sesgan los parámetros estimados llevando, en muchos casos, a la conclusión errónea de que existen rendimientos constantes a escala. Por otro lado, la utilización de la forma funcional Cobb-Douglas sesga los parámetros estimados hacia abajo si no se tiene en cuenta la heterogeneidad no observada. Este tipo de conclusiones metodológicas no han sido del todo incorporadas a la literatura aplicada a otros países (ver Ibourk et al. 2001)

En los trabajos anteriores, los coeficientes para desempleo son siempre mayores que los coeficientes para vacantes que, muchas veces no son significativos. En general, los resultados obtenidos hacen dudar de la calidad de los datos sobre vacantes. El problema de la medición de vacantes se encuentra desde la literatura más temprana y en todos los países. Aunque el registro de vacantes sea obligatorio, muchas veces las vacantes no se registran porque no tienen los mismos incentivos que los desempleados que no pueden cobrar prestaciones sin registrarse (ver Pissarides 1986 y Pedraza 2007). En este sentido, la investigación futura necesita de la obtención de datos de mayor calidad sobre vacantes. Siguiendo con aspectos relacionados con la falta de datos, y en relación con las conclusiones obtenidas en cuanto al papel que juegan en el proceso de emparejamiento aquellos que ya tienen trabajo, también sería deseable disponer de datos sobre la cantidad de personas empleadas que buscan trabajo.

El efecto agregado de las PAMT sigue siendo difícil de medir; el efecto positivo de la educación es más claro entre educación secundaria y primaria que entre secundaria y universitaria; la estructura económica de la unidad de observación, sea región o distrito, influye en 
el proceso de emparejamiento y estar más cerca de Alemania tiene un claro efecto positivo. Una estructura económica diversificada tiene un efecto positivo en la eficiencia del proceso de emparejamiento y una población activa con mayor nivel educativo también. Por tanto, el desajuste de las economías en transición tiene un perfil educacional, geográfico y estructural. Hay evidencias de que la población checa con, al menos, educación secundaria ha sido bastante flexible en cambiar de unos sectores a otros. Por último, las prestaciones por desempleo han jugado un importante papel como fuente de financiación de pequeños negocios que han permitido aumentar el flujo de salida del desempleo.

En lo que se refiere a las comparaciones entre los dos países que formaban Checoslovaquia, se encuentran diferencias tanto en los parámetros estimado de la función de emparejamiento como en el resto de variables que se han ido introduciendo como variables explicativas. Por tanto, a pesar de tener instituciones y marcos legislativos semejantes, existen diferencias entre ambas repúblicas, no sólo en cuanto al nivel de paro, sino al funcionamiento del proceso de emparejamiento.

Los últimos trabajos han señalado como líneas de investigación futura, por un lado, el cálculo del efecto del nivel educativo de los desempleados (Profit 1999). Por otro lado, la medición de los distintos factores que influyen en el fuerte efecto negativo del desempleo de larga duración en la eficiencia del emparejamiento (Pedraza 2007), ya que, mientras unos han señalado al papel desincentivador de las prestaciones sociales intensificado por la posibilidad de combinar dichas prestaciones con un trabajo en el sector informal de la economía, otros han apuntado a la importancia del nivel educativo y el desgaste del capital humano. Ambas líneas garantizan que la República checa va a seguir siendo un laboratorio para el estudio de la función de emparejamiento. Además, esta función ha sido y sigue siendo aplicada a muchos países, confirmando su utilidad como herramienta para el análisis del funcionamiento de los mercados de trabajo tanto en países en desarrollo, sufriendo cambios estructurales, como en países desarrollados en los que dichos mercados son cada vez más cambiantes y en los que cambios de un trabajo a otro, pasando o no por el desempleo, son cada vez más habituales.

\section{Conclusiones}

En primer lugar, el repaso de la literatura de la función de emparejamiento Checa es muy útil, no sólo para los estudiosos de las 
economías en transición, sino para cualquier economista laboral que se proponga estimar dicha función para cualquier otra economía. Del estudio de los trabajos que aquí se han repasado, se pueden obtener conclusiones relacionadas con distintos métodos de estimación, formas funcionales y especificaciones de la función matching incluyendo otras variables además de desempleo y vacantes.

En segundo lugar, existe una relación clara entre los estudios de duración del desempleo y aquellos que estiman funciones de emparejamiento agregadas: El efecto positivo de variables como el nivel educativo es claro mientras que el efecto negativo de la duración del desempleo y el impacto del desempleo de larga duración también. Como señalan Petróngolo y Pissarides (2001), aunque no ha sido muy habitual en la literatura, los trabajos sobre las probabilidades individuales de abandonar el desempleo pueden ser utilizados como microfundamentos a la hora de introducir nuevas variables explicativas en la función de emparejamiento agregada.

En tercer lugar, existen evidencias empíricas para dudar del supuesto de rendimientos constantes a escala, al menos, en el caso de la República Checa. Nuevos desarrollos teóricos bajo el supuesto de rendimientos crecientes son necesarios.

En cuarto lugar, la falta de datos, o la baja calidad de estos, sobre variables como vacantes y empleados inmersos en la búsqueda de trabajo, limita las conclusiones que la estimación de la función de emparejamiento permite obtener.

\section{Bibliografía}

Abraham, K. y Vodopivec (1993), "Slovenia: a study of labour market transitions", Mimeo (The World Bank, Washington, DC)

Aghion, P., y Blanchard, O.J.(1993), "On the Speed of Transition in Eastern Europe”, EBRD, Working Paper, 6 July.

Bellmann, Lutz, Saul Estrin, Hartmut Lehmann y Jonathan Wadsworth (1995), "The Eastern German labour market in transition: gross flow estimate from panel data", Journal for Comparative Economics 20, 139-170.

Blanchard, O. y P. Diamond (1989), "The Beveridge Curve", Brookings Papers on Economic Activity, 1(1989), 1-60. 
Boeri (1994), "Labour Market Flows and Persistence of Unemployment in Central Eastern Europe", Unemploymnet in Transition Countries: Transient or Persistent 13-56, Paris OCDE ed.

Boeri y Burda (1996), "Active Labour Market Policies, Job Matching and the Czech Miracle", European Economic Review Papers and Proceedings 40:805-818.

Boeri y Scarpetta (1995), "Emerging Regional Labour Market Dynamics in Central and Eastern Europe", The Regional Dimension of Unemployment in Transition Countries, 75-87, Paris OCDE ed.

Burda, M. (1993), "Unemployment, Labour markets and structural change in Eastern Europe", Economic Policy 16: 101-138.

Burda y Lubyova (1995), "The impact of Active Labour Market policies: A closer at the Czech and Slovak republics", Tax and Benefit Reforms in Central Eastern Europe, D.Newbery, ed. (CEPR London).

Burda y Profit (1996), "Matching Across Space: evidence on Mobility in Czech Republic", Labour Economics 3, 255-278.

Coles, M.G. y Smith E. (1994), "Market Places and Matching" CEPR Discussion paper No. 1048.

Coles, M.G. y Smith E. (1998), "Market Places and Matching", International Economic Review 39, 239-354.

Dushi, I. (1998), "Labour market, unemployment and the impact of training programs in the transition economies", Mimeo, CERGE-EI Doctoral Dissertations, Prague.

Finta, Jana y Katherine Terrell (1997), "Gender differences in flows across labour market states in the Czech Republic", Trabajo no publicado, University of Michigan, Ann Arbor, MI.

Foley, M.C. (1997), "Determinants of unemployment duration in Russia”, William Davidson working paper no.81, University of Michigan Business School, Ann Arbor, MI. 
Gross, D.M. (1997), "Aggregate job matching and returns to scale in Germany” Economics Letters 56: 243-8.

Ham, J., J. Svejnar y K. Terrell (1998), "Unemployment and the social safety net during transitions to a market economy: evidence from the Czech and Slovak Republics", American Economics Review 88 (5): 1117 1142 .

Ham, J., J. Svejnar y K. Terrell (1999), “Women's unemployment during transition: evidence from Czech and Slovak micro-data", Economics of Transition 7(1):47-78.

Ibourk A., B. Maillard, S. Perelman y H.R. Sneessens (2001), "The Matching Efficiency of Regional Labour Markets: A Stochastic Production Frontier Estimation, France 1990-1995, IZA Discussion paper no. 339, Germany.

Jones, Derek C. y Takao Kato (1997), "The nature and determinants of labour market transitions in former communist economies: evidence from Bulgaria", Industrial Relations 36 (2): 229-254.

Jurajda S. y K. Terrell (2002a), "What Drives the Speed of Job Reallocation During Episodes of Massive Adjustment" William Davidson Institute Working paper no.432

Jurajda S. y K. Terrell (2002b), "Job Growth in Early Transition: Comparing Two Paths" William Davidson Institute Working paper no. 434

Jurajda S. (2005), "Are there increasing Returns to local Concentration of Skills? Evidence on Wages and Returns to Education in Transition ". CERGE-EI Working paper 260, 2005

Layard, Richard, S. Nickell y R. Jackman (1991), Unemployment: Macroeconomic performance and the labour market. Oxford University Press.

Lubyova, Martina y van Ours (1994), "The Matching Process in Labour Markets in Transition", Working paper no.13 East European HIS Vienna. 
Lubyova, Martina y van Ours (1997), "Work incentives and the probability of leaving unemployment in Slovak Republic", William Davidson working paper no.82, University of Michigan Business School, Ann Arbor, MI.

Micklewright, J. y G. Nagy (1997), "The implications of exhausting unemployment insurance entitlement in Hungary" Occasional paper no. 58 (Economic and Social Policy Series, United Nations Children's fund).

Munich et al. (1998). The worker firm matching in transition: (why) are Czech more successful than others? William Davidson Institute Working paper no.82, University of Michigan Business School, Ann Arbor, MI.

Munich y Golusčak (2003). Microfundations of wage inflation in the Czech Republic. Czech National Bank Working Paper Series 1/03. Disponible en http://www.cnb.cz

Pedraza P. (2007), "The matching function of a Transition Economy: The case of the Czech Republic", Tesis doctoral no publicada, disponible en http://tusalario.es/main/queestusal/equipotusal2607

Petrongolo B. y C.A. Pissarides (2001), "Looking into the black box: A survey of the matching function", Journal of Economic Literature vol. XXXIX (June) pp. 390-431.

Pissarides, C.A. (1986), "Unemployment and Vacancies in Britain", Economic Policy 3, pp. 457-75.

Pissarides , C.A.(1992) Equilibrium Unemployment Theory, MIT first ed.

Pissarides, C.A.(2000) Equilibrium Unemployment Theory, MIT second ed.

Profit (1996), "Unemployment Dynamics and Returns to Scale in Job Matching in Emerging Labour Markets: The Case of the Czech Republic", mimeo, Humboldt University, Berlin.

Profit (1999), "Twin Peaks in Regional Unemployment and Retursn to Scale in job Matching in the Czech republic", Discussion Paper $n^{\circ}$ 63, Humboldt University, Berlin. 
Profit y Sperlich (2004), "Non-Uniformity of Job-Matching in a Transition Economy - A non-parametric Analysis of the Czech Republic", Applied Economics 36: 695-714.

Ronald, G. (2000). Transition and Economics: Politics, Markets, and Firms. Cambridge, MA, 2000.

Sorm, V. y K. Terrell (1997), "Employment, unemployment in transition in the Czech Republic: where have all the workers gone?" Mimeo (William Davidson Institute, University of Michigan Business School, Ann Arbor, MI)

Sorm, V. y K. Terrell. (1998), "Labour Market Policies and Unemployment in the Czech Republic", The William Davidson Institute Working Paper no. 216

Sorm, V. y K. Terrell (1999a), "A Comparative Look at Labour Mobility in the Czech Republic: Where Have All the Workers Gone?" The William Davidson Institute Working Paper no. 140

Sorm, V. y K. Terrell. (1999b), "Sectoral Restructuring and Labour Mobility: A Comparative Look at the Czech Republic", The William Davidson Institute Working Paper no. 273

Svejnar, J., K.Terrell and D. Munich (1995), "Unemployment in the Czech and Slovak Republics", en Svejnar (1995) pp. 285-316.

Svejnar, Jan (1995), The Czech Republic and Economic Transformation of Eastern Europe, San Diego; London and Toronto: Harcour Brace, Academic Press.

Svejnar, Jan (1999), "Labour Markets in the Transitional Central and East European Economies", capítulo de O. Ashenfelter and D. Card (eds.), Handbook of Labour Economics, volume 3, 2809-2857. 


\section{Anexo I. Cuadro resumen}

\begin{tabular}{|c|c|c|c|c|}
\hline Autor & Datos & $\begin{array}{l}\text { Especifica } \\
\text { ción }\end{array}$ & Estimación & Conclusiones \\
\hline $\begin{array}{l}\text { Burda } \\
\text { (1993) }\end{array}$ & $\begin{array}{l}\text { Mensuales de } \\
\text { distritos } \\
\text { checos }\end{array}$ & $\begin{array}{c}\text { Cobb - } \\
\text { Douglass }\end{array}$ & Panel MCO & $\begin{array}{l}\text {-Existencia de una función de } \\
\text { emparejamiento estable } \\
\text {-Rendimientos constantes }\end{array}$ \\
\hline Boeri (1994) & $\begin{array}{l}\text { Anuales de } \\
\text { regiones }\end{array}$ & $\begin{array}{c}\text { Cobb - } \\
\text { Douglass }\end{array}$ & $\mathrm{MCO}$ & $\begin{array}{l}\text {-Rendimientos decrecientes } \\
\text { constantes } \\
\text {-El impacto del paro de larga } \\
\text { duración es menor }\end{array}$ \\
\hline $\begin{array}{l}\text { Svejnar et } \\
\text { al. (1995) }\end{array}$ & Anuales & $\begin{array}{c}\text { Cobb - } \\
\text { Douglass }\end{array}$ & $\begin{array}{c}\text { Sección cruzada } \\
\text { MCO }\end{array}$ & $\begin{array}{l}\text {-Coeficientes para desempleo son el } \\
\text { doble que los de vacantes y mayores } \\
\text { en la República Checa que en la } \\
\text { Eslovaca. } \\
\text {-Efecto positivo de PAMT en Chequia } \\
\text { y no significativo en Eslovaquia }\end{array}$ \\
\hline $\begin{array}{l}\text { Lubyova y } \\
\text { van Ours } \\
(1994)\end{array}$ & $\begin{array}{l}\text { Mensuales de } \\
\text { regiones }\end{array}$ & $\begin{array}{l}\text { Cobb - } \\
\text { Douglass } \\
\text { para cada } \\
\text { región }\end{array}$ & $\mathrm{MCO}$ & -Rendimientos crecientes \\
\hline $\begin{array}{l}\text { Boeri y } \\
\text { Scarpetta } \\
\text { (1995) }\end{array}$ & $\begin{array}{l}\text { Mensuales } \\
\text { distritos }\end{array}$ & $\begin{array}{c}\text { Cobb - } \\
\text { Douglass }\end{array}$ & $\mathrm{MCO}$ & -Rendimientos decrecientes \\
\hline $\begin{array}{l}\text { Burda } \quad y \\
\text { Lubyova } \\
\text { (1995) }\end{array}$ & $\begin{array}{l}\text { Mensuales y } \\
\text { Trimestrales }\end{array}$ & $\begin{array}{c}\text { Cobb - } \\
\text { Douglass }\end{array}$ & $\begin{array}{l}\text { Desviaciones } \\
\text { respecto a la } \\
\text { media }\end{array}$ & $\begin{array}{l}\text {-Coeficientes para desempleo muy } \\
\text { altos } \\
\text {-Las PAMT tienen un efecto positivo }\end{array}$ \\
\hline $\begin{array}{l}\text { Boeri } \\
\text { Burda } \\
(1996)\end{array}$ & $\begin{array}{l}\text { Mensuales y } \\
\text { Trimestrales } \\
\text { de distritos }\end{array}$ & $\begin{array}{c}\text { Cobb - } \\
\text { Douglass }\end{array}$ & $\begin{array}{l}\text { Desviaciones } \\
\text { respecto a la } \\
\text { media }\end{array}$ & $\begin{array}{l}\text {-Las PAMT tienen un efecto positivo } \\
\text { pero pequeño } \\
\text {-Rendimientos } \\
\text { decrecientes }\end{array}$ \\
\hline $\begin{array}{lr}\text { Burda y } & \text { y } \\
\text { Profit (1996) }\end{array}$ & $\begin{array}{l}\text { Regionales y } \\
\text { de distritos }\end{array}$ & $\begin{array}{c}\text { Cobb - } \\
\text { Douglass }\end{array}$ & $\mathrm{MCO}$ & $\begin{array}{ll}\text {-No desechan la existencia de } \\
\text { rendimientos constantes } & \\
\text {-Creciente importancia de los } \\
\text { pequeños negocios y el desajuste }\end{array}$ \\
\hline Profit (1996) & $\begin{array}{l}\text { Mensuales de } \\
\text { distritos }\end{array}$ & $\begin{array}{c}\text { Cobb - } \\
\text { Douglass }\end{array}$ & $\begin{array}{c}\text { Variables } \\
\text { instrumentales } \\
\text { Anderson-Hsiao }\end{array}$ & $\begin{array}{l}\text { Utilizando VI Anderson-Hsiao, para } \\
\text { resolver autocorrelación y } \\
\text { heterocedasticidad, la función de } \\
\text { emparejamiento checa despliega } \\
\text { rendimientos crecientes a escala. }\end{array}$ \\
\hline $\begin{array}{l}\text { Munich et } \\
\text { al. (1998) }\end{array}$ & $\begin{array}{l}\text { Mensuales de } \\
\text { distritos }\end{array}$ & Translog & $\begin{array}{l}\text { Desviaciones } \\
\text { respecto medias } \\
\text { hacia atrás. }\end{array}$ & $\begin{array}{l}\text {-Rendimientos a escala crecientes por } \\
\text { las altas elasticidades del desempleo } \\
\text {-Efecto negativo distancia } \\
\text {-Efecto no significativo PAMT } \\
\text {-Efecto ambiguo de niveles educativos } \\
\text { y producción agrícola e industrial } \\
\text {-Efecto negativo paro de larga } \\
\text { duración }\end{array}$ \\
\hline
\end{tabular}




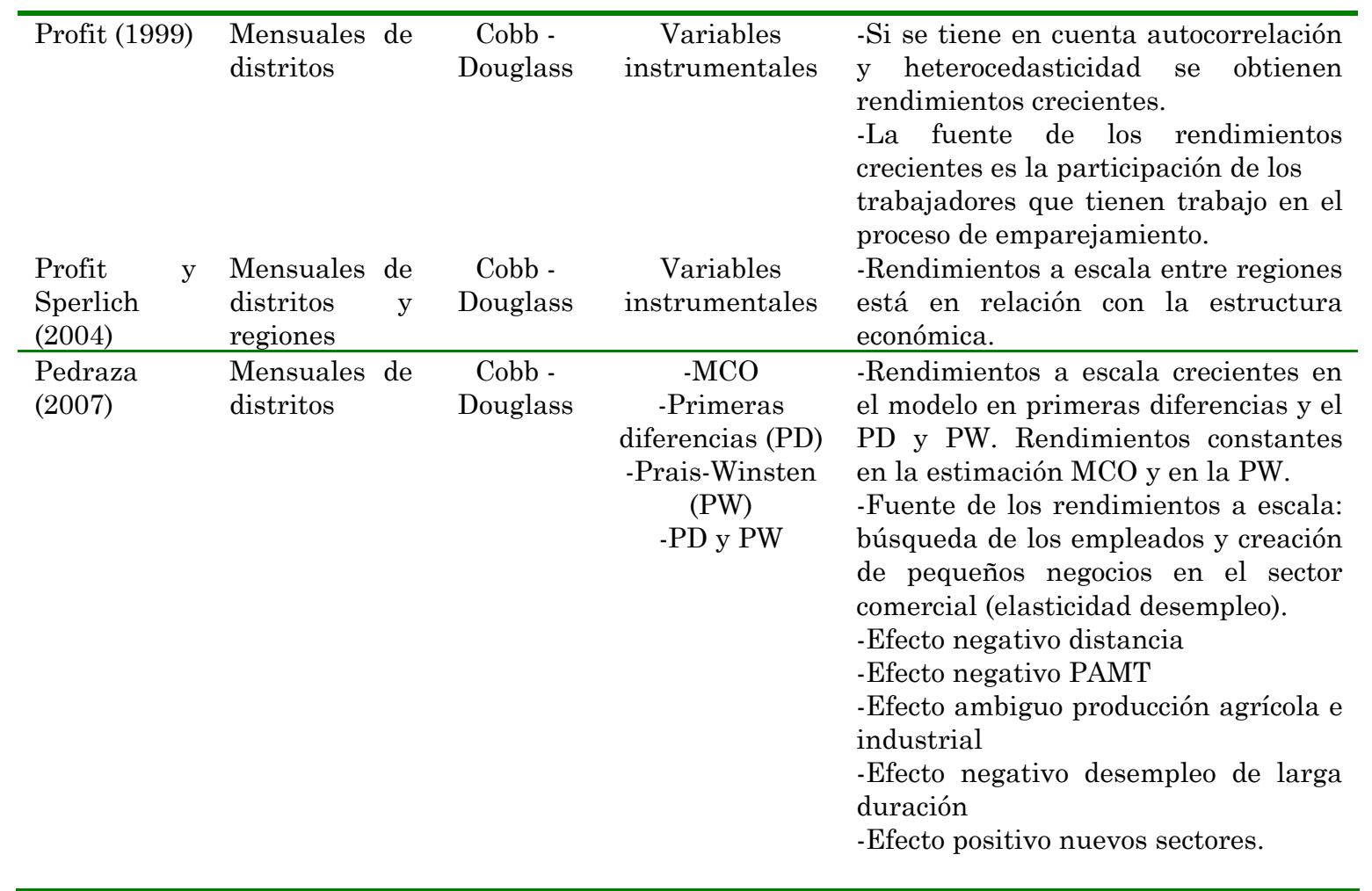

